\title{
Influence of Primary and Secondary Crystallographic Orientations on Strengths of Nickel-based Superalloy Single Crystals
}

\author{
Koji Kakehi \\ Department of Mechanical Engineering, Tokyo Metropolitan Institute of Technology, Hino 191-0065, Japan
}

It has been revealed that the strengths of the notched specimens were affected by the crystallographic orientation not only in the tensile direction (primary orientation) but also in the thickness direction (secondary orientation). In this study, by using single crystals of an experimental superalloy which shows distinct active slip systems, the influence of primary and secondary crystal orientation on creep and fatigue strengths of Ni-based superalloy single crystals was investigated. The influence of crystallographic orientations and plastic anisotropy on the creep and fatigue strengths of single crystals of the Ni-base superalloy was discussed on the assumption that $\{111\}\langle 101\rangle$ and $\{111\}\langle 112\rangle$ slip systems. $\{111\}\langle 112\rangle$ slip is unusual slip. For a tensile stress applied close to the [001] axis, (111)[1 12$]$ slip system is result of pass of intrinsicextrinsic superlattice stacking faults pair through the $\gamma^{\prime}$ phase. In the tensile orientation close to [011], (111)[211] slip is occurred by twinning shear through $\gamma$ and $\gamma^{\prime}$ phases. In the case of creep strength, the results were in agreement with the assumption of the operation of the $\{111\}\langle 112\rangle$ slip in the primary creep and $\{111\}\langle 101\rangle$ slip in the secondary creep. The notched creep behavior was found to be influenced by the additional aging at $850^{\circ} \mathrm{C}$ for $20 \mathrm{~h}$, which prohibited activity of $\{111\}\langle 112\rangle$ slip systems. The fatigue lifetime and crack growth behavior depended on both plastic anisotropy caused by arrangement of $\{111\}\langle 101\rangle$ slip systems and the stress state.

(Received February 19, 2004; Accepted April 8, 2004)

Keywords: nickel-based superalloy, single crystals, primary and secondary crystallographic orientations, creep, fatigue, heat treatment, plastic anisotropy

\section{Introduction}

Ni-based superalloy single crystals are used for turbine blade of aircraft jet engines because of their excellent creep, stress rupture, thermo mechanical fatigue capabilities. Microstructure of a Ni-based superalloy single crystal consists of a $\gamma$ phase and cuboidal $\gamma^{\prime}$ precipitates, whose interfaces are parallel to the $\{001\}$ planes. A dendrite structure grows in the $\langle 001\rangle$ direction in the face-centered-cubic Ni-based superalloy. This orientation provides better creep strength and a low modulus of elasticity that enhances thermal fatigue resistance. Thus, the turbine blades are designed so that their primary orientation is within 10 to $15^{\circ}$ of the $\langle 001\rangle$ axis to insure better creep strength and thermal fatigue resistance. The secondary dendrite direction $(\langle 010\rangle$ direction $)$ is usually randomly oriented with respect to the longitudinal direction of the turbine blade. The air-cooled turbine blades, which have a complicated hollow structure, are composed of sections of various thicknesses. Therefore, the mechanical properties of each blade section will depend on plastic anisotropy and the stress state as well as stress in the longitudinal direction.

Fatigue crack growth rate was affected by the plastic anisotropy in the crack tip due to the geometric arrangement of the $\{111\}$ slip planes. ${ }^{1,2)}$ Since a single crystal possesses the intrinsic plastic anisotropy, the strengths of single crystals are influenced by the crystallographic orientations not only in the tensile direction but also in the normal direction of the specimen. ${ }^{3-5)}$ The important point to note is that optimization of secondary crystallographic orientation (angle of the [100] orientation and airfoil mean chord line) has the potential to significantly increase a turbine blade's resistance to fatigue crack growth without additional weight or cost. ${ }^{6}$ ) Although a large number of studies have been made on effect of primary orientation, little is known about that of secondary orientation. ${ }^{6)}$ In this study, by using the single crystals of the experimental superalloy which shows distinct active slip systems, ${ }^{7)}$ the influence of secondary orientation on the creep and fatigue strengths was investigated.

\section{Experimental Procedures}

The chemical composition of the Ni-based superalloy is listed in Table 1. Single crystals of this alloy were grown from the melt by the modified Bridgman method. After the analysis of the crystallographic orientation by the back Laue reflection method, notched creep $^{4)}$ and fatigue (Fig. 1) specimens were cut out from the as-grown crystals using a spark cutter. Thicknesses of the notched specimen were determined to obtain the plane stress condition. ${ }^{3)}$ Crystallographic orientations of the four kinds of specimens with

Table 1 Chemical composition (mass \%).

\begin{tabular}{cccccccc}
\hline Cr & Mo & Co & W & Ti & Al & V & Ni \\
\hline 10.1 & 2.50 & 9.97 & 0.04 & 4.75 & 5.73 & 0.93 & Bal.
\end{tabular}

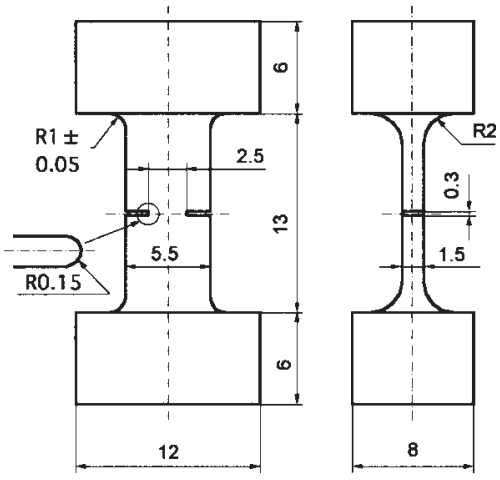

Fig. 1 Notched fatigue specimen (mm). 
(a)

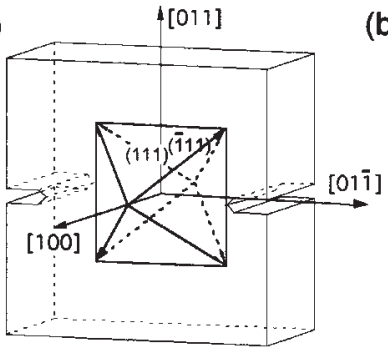

(c)
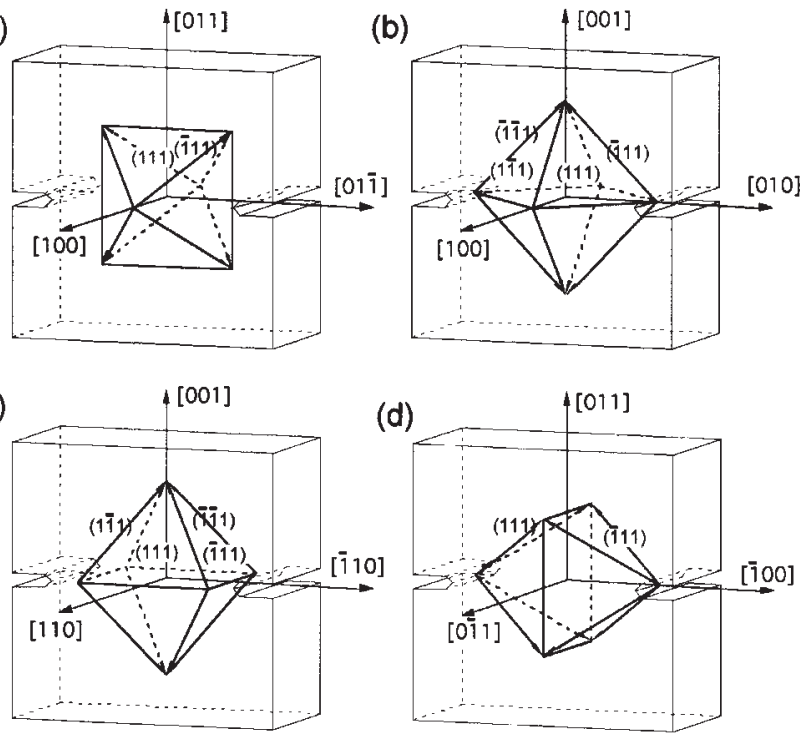

(d)

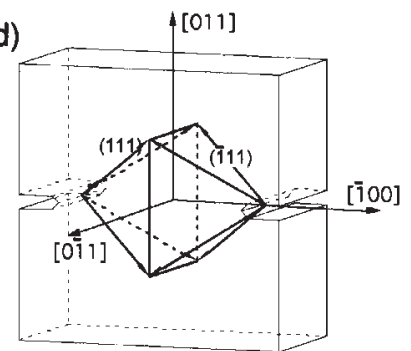

Fig. 2 Four kinds of specimens and arrangement of octahedral slip systems; (a) orientation A, (b) orientation B, (c) orientation C and (d) orientation D.

different arrangements of slip systems are shown in Fig. 2. The specimens were subjected to solution treatment at $1255^{\circ} \mathrm{C}$ for $10 \mathrm{~h}$, and to aging treatment at $1100^{\circ} \mathrm{C}$ for $10 \mathrm{~h}$ and subsequently to an additional aging at $850^{\circ} \mathrm{C}$ for $20 \mathrm{~h}$. After each heat treatment, the specimens were cooled in an air-blast. A single-step aging treatment at $1100^{\circ} \mathrm{C}$ for $10 \mathrm{~h}$ resulted in the clear $\{111\}\langle 112\rangle$ slip because of numerous hyperfine secondary $\gamma^{\prime}$ precipitates in the matrix channel; ${ }^{7)}$ therefore, the single-step aging treatment was also employed for creep specimens. The average edge lengths were $0.40 \mu \mathrm{m}$ for the single-aged specimen and $0.39 \mu \mathrm{m}$ for the doubleaged specimen, respectively.

Creep and fatigue rupture tests were conducted in laboratory environmental and at $700^{\circ} \mathrm{C}$. Creep rupture test was carried out under a nominal stress of $820 \mathrm{MPa}$ for the rectangular-cross-section specimens. In the case of the notched specimens, the stress was equally applied for the initial net cross-sectional area. The tension-zero fatigue test (the stress range of $600 \mathrm{MPa}$ was loaded for the net crosssectional area) was done at a frequency of $5 \mathrm{~Hz}$.

\section{Experimental Results}

\subsection{Creep rupture test}

The creep properties of rectangular-cross-section specimens are shown in Table 2. The rectangular-cross-section

Table 2 Creep properties of rectangular cross-section specimens $\left(700^{\circ} \mathrm{C}\right.$, $820 \mathrm{MPa})$.

\begin{tabular}{cccc}
\hline Specimen & $\begin{array}{c}\text { Primary creep } \\
\text { strain (\%) }\end{array}$ & $\begin{array}{c}\text { Rupture life } \\
(\mathrm{h})\end{array}$ & $\begin{array}{c}\text { Rupture } \\
\text { elongation (\%) }\end{array}$ \\
\hline [001] single-aged & 6.0 & 520.6 & 10.1 \\
[001] double-aged & 1.5 & 385.1 & 3.85 \\
[011] single-aged & - & 5.6 & 23.5 \\
[001] double-aged & - & 4.8 & 10.0 \\
\hline
\end{tabular}
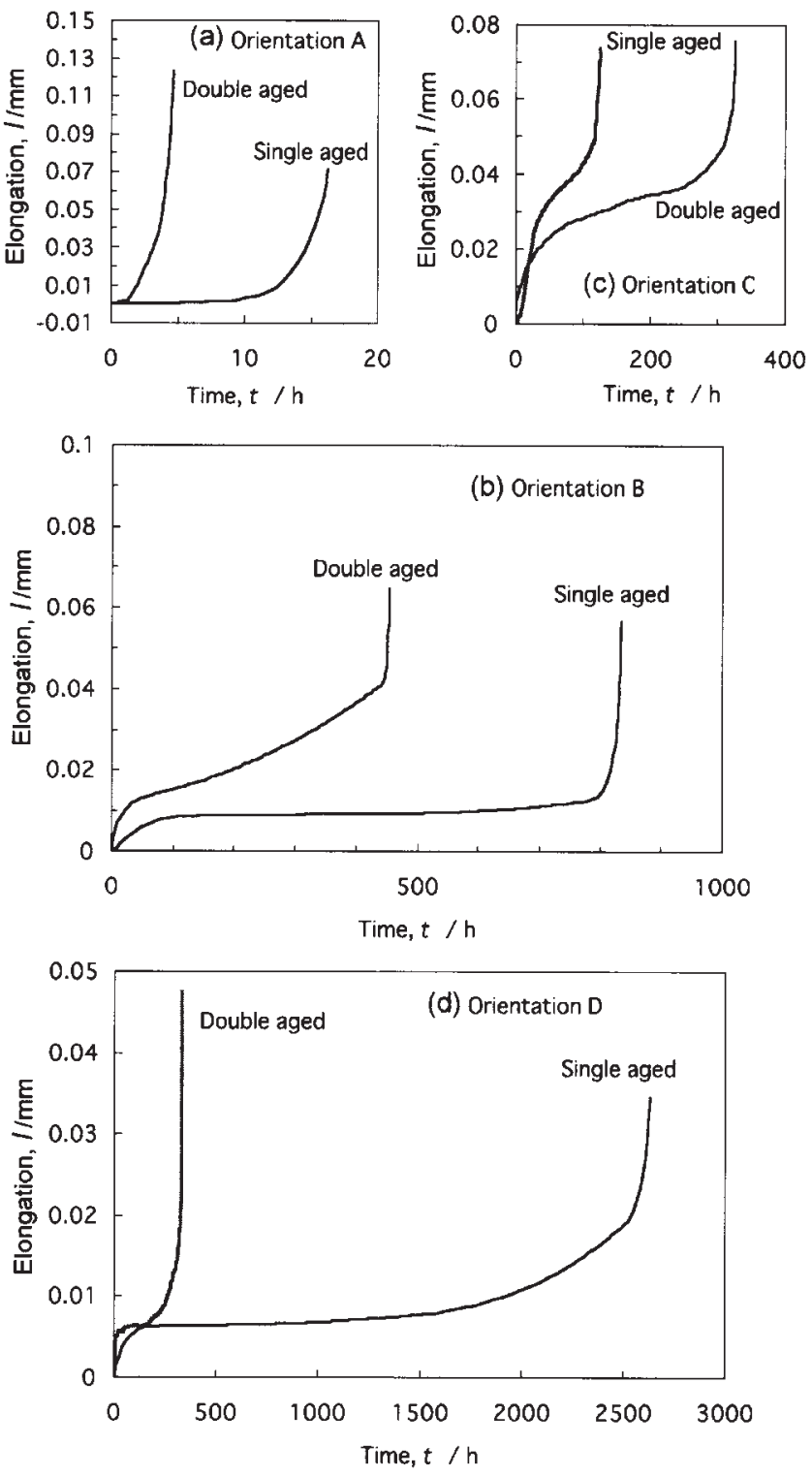

Fig. 3 Influence of aging heat treatment on creep curves in four kinds of notched specimens.

specimen with the [001] orientation showed large primary creep strain of $6.0 \%$ and steady-state creep. The [011] orientation exhibited appreciably shorter lives and large rupture elongation because of the absence of strain hardening. In the case of the notched creep, single-aged specimens showed distinct plastic anisotropy. ${ }^{4)}$ When the tensile direction is [001], the rupture lifetime of orientation B was seven times longer than that of orientation C. Creep curves of the notched specimens are shown in Fig. 3. In the case of specimens whose tensile orientation is [011], in spite of the poor creep strength of the rectangular-cross-section specimen (Table 2), orientation D exhibited extremely small steadystate creep rate and exceptionally long rupture time. Conversely, orientation A showed larger creep rate and shorter rupture lifetime than orientation D. The rupture lifetime of orientation D was 162 times longer than that of orientation A. In the single-aged specimens, it is obvious that the notched-tensile creep strength is influenced by the crystallographic orientations not only in the tensile direction 


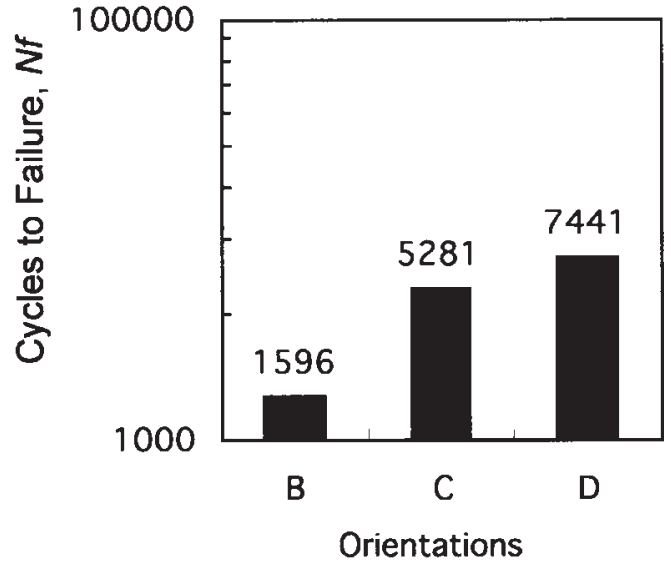

Fig. 4 Orientation dependence of fatigue rupture lifetime.

but also in the thickness direction.

The creep behavior was found to be influenced by the additional aging at $850^{\circ} \mathrm{C}$ for $20 \mathrm{~h}$. As shown in Fig. 3(a), in orientation $\mathrm{A}$, the single-aged specimen showed an incubation period, but the incubation period disappeared in the specimen aged at $850^{\circ} \mathrm{C}$. Both specimens exhibited very short the rupture lifetime. The creep rupture lifetime was increased by the second aging at $850^{\circ} \mathrm{C}$ in orientation $\mathrm{C}$. However, both in orientations $\mathrm{B}$ and $\mathrm{D}$, the creep rupture lifetime was decreased by the second aging at $850^{\circ} \mathrm{C}$.

\subsection{Fatigue test}

Crystallographic orientation dependence of fatigue rupture lifetime is presented in Fig. 4. Orientation D showed the longest fatigue lifetime. Orientations $\mathrm{B}$ and $\mathrm{C}$ have same tensile [001] direction; however, orientation $\mathrm{C}$ showed longer fatigue lifetime than orientation B. Figure 5 showed fatigue fracture surfaces. Stage I crack and mode I-dominated stage II crack growth regions were observed. As shown in Fig. 6, as the area fraction of stage II region increased, the fatigue lifetime decreased. Stage II crack propagation took up much of the fatigue lifetime of the thin notched specimens.

\section{Discussion}

Single-aged specimens showed distinct active $\{111\}\langle 112\rangle$ slip systems and plastic anisotropy. ${ }^{7}$ The creep rupture test results of single-aged specimens were in agreement with the assumption of the operation of $\{111\}\langle 112\rangle$ slip systems during primary creep region and $\{111\}\langle 101\rangle$ slip systems during secondary creep region. ${ }^{4)}$ The $\{111\}\langle 112\rangle$ slip systems in four kinds of specimens are illustrated in Fig. 7. The group of slip systems that results in a contraction of the thickness of the specimen is named "T group", while the group of slip systems that results in a contraction of the width of the specimen is named "W group", which necessarily accompanies the contraction in the width direction and would be constrained. If a group of slip systems results in a contraction of both the thickness and width of the specimen, it is named "TW group". In orientation B, even under a complex multiaxial stress state, a lateral distortion in the thickness direction is simultaneously produced with that in the width direction. Even if only one slip system operates, the contraction in the thickness direction necessarily accompanies that in the width direction, therefore, the slip systems belonging to the TW group would be constrained. Singe-aged specimen of orientation B has no unconstrained $\{111\}\langle 112\rangle$ systems but $\{111\}\langle 101\rangle$ slip systems belonging to the $\mathrm{T}$ group (Fig. 2(b)); therefore, it would rapidly enter into secondary creep region as shown in Fig. 3(b). Whereas, in orientation $\mathrm{C}$, as shown in Fig. 7(c), there are two kinds of slip systems. The slip systems belonging to $\mathrm{W}$ group would be constrained; however, those belonging to $\mathrm{T}$ group would not be constrained. The poor creep resistance of orientation $\mathrm{C}$ (Fig. 3(c)) resulted from a combination of the free operation of two $\{111\}\langle 112\rangle$ slip systems combined with a lack of activity on the $\{111\}\langle 101\rangle$ slip systems which would terminate primary creep. In orientation A, none of the slip systems belonging to T group (Figs. 2(a) and 7(a)) could be constrained and the creep strengths were extremely low.

The creep behavior was found to be greatly influenced by the additional aging at $850^{\circ} \mathrm{C}$ for $20 \mathrm{~h}^{7}{ }^{7}$ In orientation $\mathrm{A}$, as shown in Fig. 3(a), the single-aged specimen showed an incubation period, but the incubation period disappeared in the specimen aged additionally at $850^{\circ} \mathrm{C}$. The hyperfine secondary precipitates would have retarded dislocation glide in the matrix channel, and brought about an incubation period in the single-aged specimen. However, the incubation period disappeared in the specimens aged at $850^{\circ} \mathrm{C}$. It is because the matrix dislocations will glide gradually in the matrix channel, bypassing the large particles, which reprecipitate during the second aging at $850^{\circ} \mathrm{C}$. The creep rupture lifetime was increased by the second aging at $850^{\circ} \mathrm{C}$ in orientation $\mathrm{C}$. The poor creep resistance of single-aged specimen of orientation $\mathrm{C}$ resulted from the free operation of the two $\{111\}\langle 112\rangle$ slip systems. As a result of the additional aging at $850^{\circ} \mathrm{C}$, the hyperfine $\gamma^{\prime}$ precipitates coarsened and the resultant large mean distance between the coarsened and cuboidal precipitates inhibited extensive shearing of the $\gamma-\gamma^{\prime}$ structure by the (111)[112] slip system. ${ }^{7)}$ Inhibition of operation of $\{111\}\langle 112\rangle$ slip systems would bring about increase of creep lifetime in the double-aged specimen. Both in orientations B and $D$, all $\{111\}\langle 112\rangle$ slip systems are constrained. However in these orientations, the creep rupture lives were decreased by the second aging at $850^{\circ} \mathrm{C}$ because the other slip systems would be activated; (1) $\{111\}\langle 101\rangle$ slip systems which shear both $\gamma$ and $\gamma^{\prime}$ phases, and (2) macroscopic cube slip by the zigzag dislocation motion in the matrix channel. ${ }^{8)}$ In orientation D, as shown in Fig. 8(b), cube slip systems belong to $\mathrm{T}$ group which is not constrained. In double-aged specimens, $\{111\}\langle 112\rangle$ slip systems which show distinct plastic anisotropy were inhibited, and isotropic deformation, consisted of the dislocation motion in matrix channel and $\{111\}\langle 101\rangle$ shear slip, would have become operative during creep. The point is that the second aging at $850^{\circ} \mathrm{C}$ decreased the extent of plastic anisotropy in creep test.

In the fatigue test, as the area fraction of stage II region increased, fatigue lifetime increased (Fig. 6). This result indicates that stage II crack propagation would take up much of the fatigue lifetime of the thin notched specimens. The transition from stage II crack propagation on (001) plane to stage I crack propagation on $\{111\}$ planes brings about increase of crack growth rate. ${ }^{9,10)}$ Telesman and Ghosn 
(a)
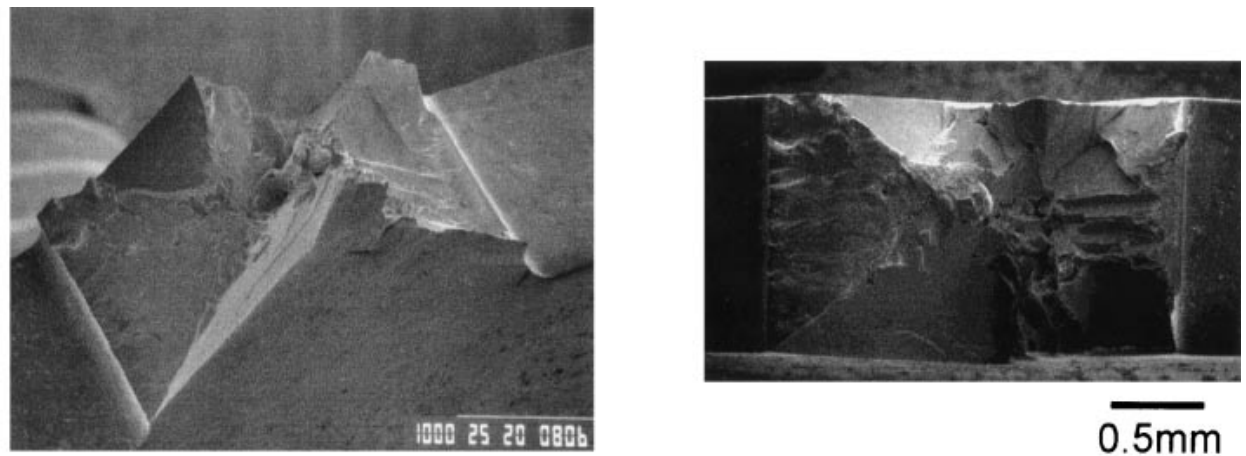

(b)
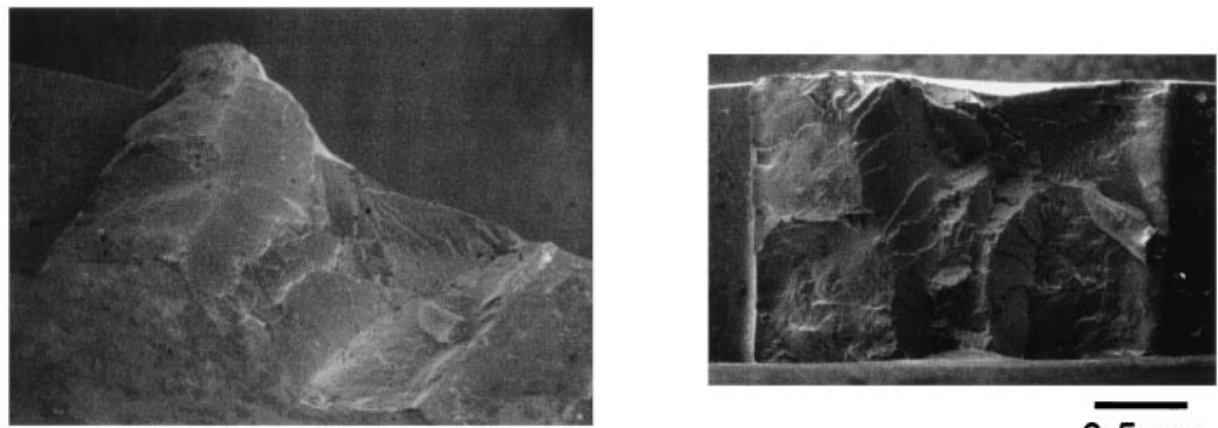

$\overline{0.5 \mathrm{~mm}}$

(c)
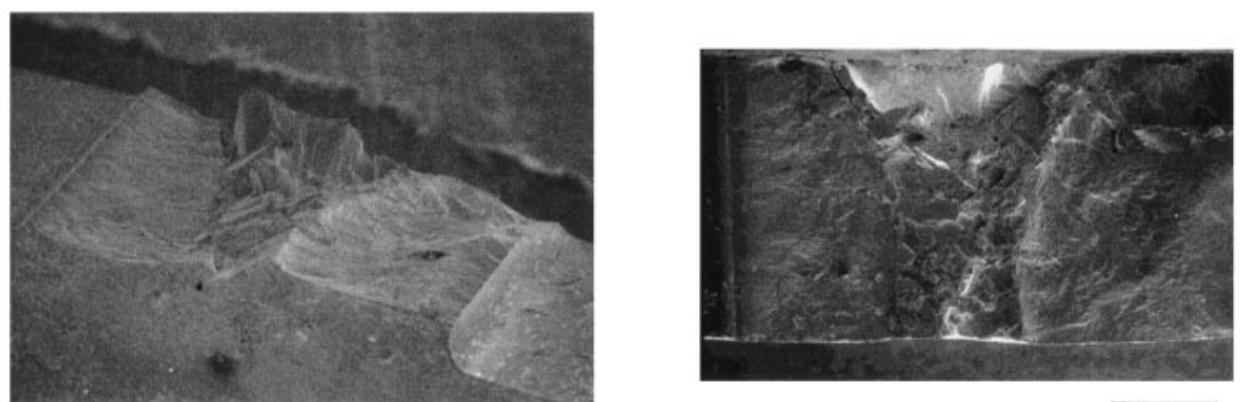

$\overline{0.5 \mathrm{~mm}}$

Fig. 5 Scanning electron fractographs showing the cleavage crystallographic fracture by slip-band decohesion and stage II crack propagation; (a) orientation B, (b) orientation C, (c) orientation D.

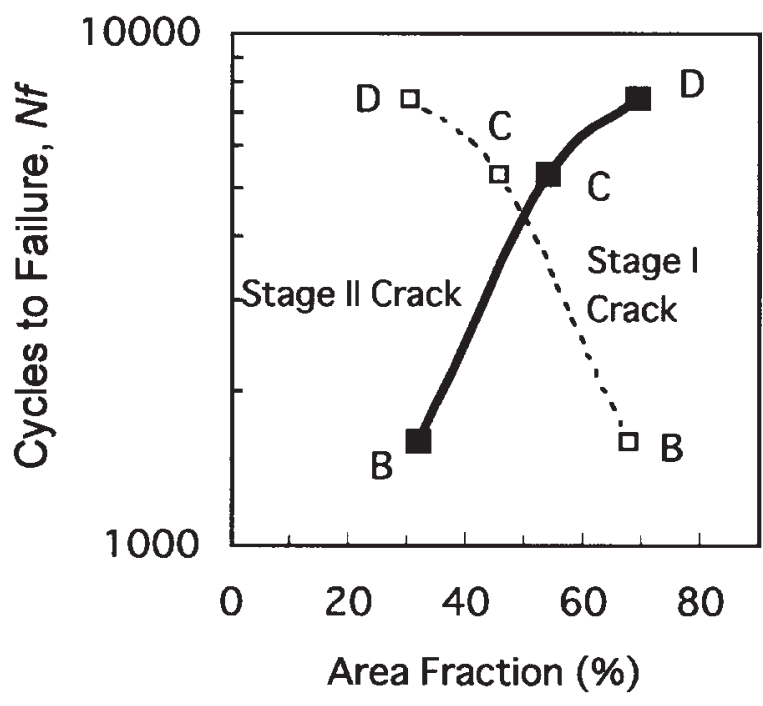

Fig. 6 Relationship between area fraction of stable crack trace on the fracture surface and cycles to rupture. showed the relationship between crack growth and the stress intensity. ${ }^{9)}$ As the stress intensity was increased, area containing facets on $\{111\}$ planes also became appearance. With a further increase in the stress intensity, the (001) fatigue failure completely disappeared, and was replaced by the $\{111\}$ fatigue failure. The increase in the $\Delta K$ resulted in an increase in the size of the $\{111\}$ failure facets.

In orientation $\mathrm{B}$, in this study, the cleavage crystallographic-fracture surface by slip-band decohesion was observed and rupture lifetime was the shortest. The reason for the result is that it is difficult to relax stress concentration at crack tip because of arrangement of $\{111\}\langle 101\rangle$ slip systems in orientation $\mathrm{B}^{5}{ }^{5}$ The secondary slip with a shear displacement component perpendicular to the crack plane can relax the normal stress of the primary slip, therefore, the difficulty of activating secondary slip in return results in large hydrostatic and normal stresses near the crack tip. ${ }^{11)}$ The combination of intense coplanar shear and large normal stresses results in easy crack propagation and low toughness. ${ }^{11)}$ However, in the orientation, under plane strain, all four slip planes were active and crack path zigzagged on two 


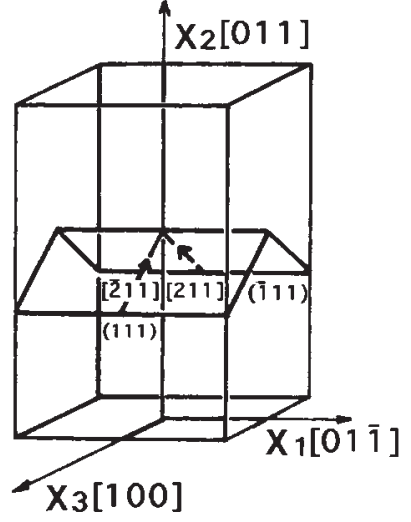

(a) Orientation A

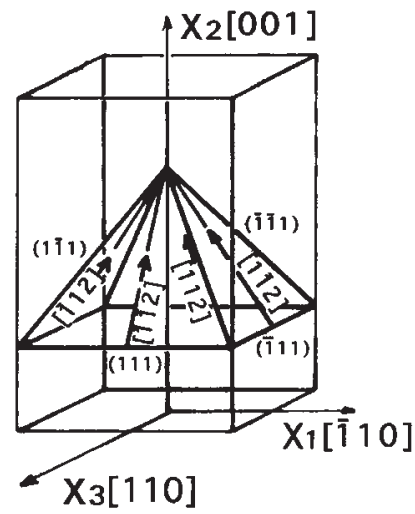

(c) Orientation C

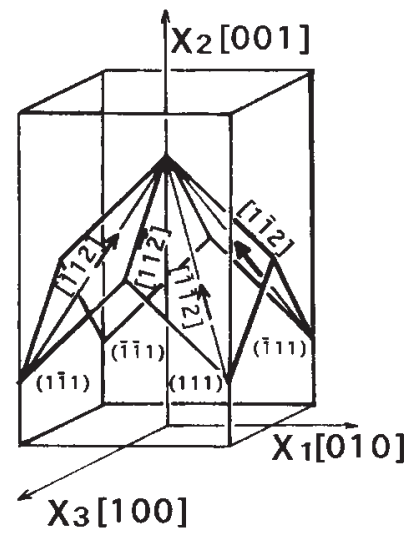

(b) Orientation B

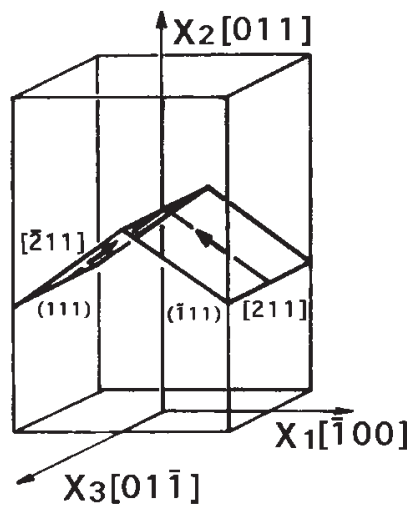

(d) Orientation D

Fig. 7 Arrangement of $\{111\}\langle 112\rangle$ slip systems for four kinds of specimens.

distinct pairs of $\{111\}$ planes. $\left.{ }^{9}\right)$ The difference in fatigue failure appearance between this study and others ${ }^{2,9)}$ would be due to the stress condition. The resolved shear stress intensity parameter (Krss) was able to predict the microscopic crack path under difference stress states, and that the magnitude of $\Delta K r s s$ under the plane stress is always approximately twice that of plane strain. ${ }^{9)}$ Compared with the thick specimens, the cleavage crystallographic-fracture surface by slip-band decohesion would easily occur in the thin specimens used in this study because of the plane stress condition.

In orientation $\mathrm{C}$, shear symmetry between $(1 \overline{1} 1)$ and $(\overline{1} 11)$ planes with respect to the cracking (001) plane would be able to relax stress concentration of the crack front. As a result of stable stage-II cracking, the fatigue lifetime was longer than that of orientation B. When the resolved shear stress is below the critical value needed for a dislocation to cut through the precipitates, the slip becomes confined in the matrix. ${ }^{9)}$ The localization of the damage to the $\{111\}$ matrix channel results in a preferential failure in this area, exposing the cuboidal facets of the precipitates and creating a (001) failure appearance.

Furthermore, in orientation D, in addition to shear symmetry between (111) and (111) slip planes with respect

(a) Orientation $\mathrm{A}$

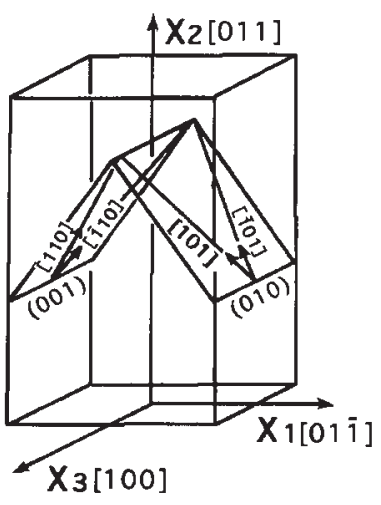

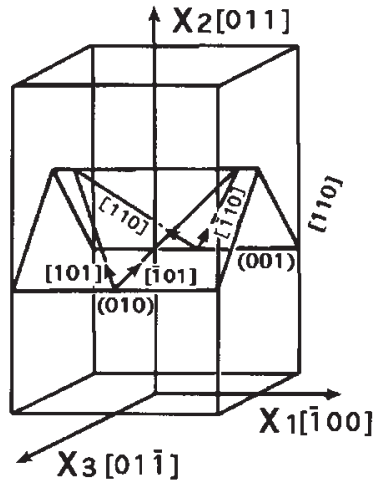

(b) Orientation D
Fig. 8 Arrangement of $\{001\}\langle 101\rangle$ slip systems for two kinds of specimens.

to the cracking (011) plane, there is no slip system belonging to $\mathrm{T}$ group, which results in cleavage crystallographicfracture surface by slip-band decohesion and short fatigue lifetime. Therefore, orientation D exhibited the largest cycles to failure among three kinds of crystallographic specimens.

\section{Conclusions}

(1) Creep and fatigue strengths of the notched-tensile specimens were influenced by the crystallographic orientations not only in the tensile direction but also in the thickness direction.

(2) Orientation D exhibited superior creep and fatigue strengths because both $\{111\}\langle 101\rangle$ and $\{111\}\langle 112\rangle$ slip systems are constrained. However operation of macroscopic cube slip decreased creep strength in this orientation.

(3) Second-step aging treatment at $850^{\circ} \mathrm{C}$ decreased the influence of plastic anisotropy on notched creep strength.

(4) The fatigue lifetime and crack growth behavior depended on both plastic anisotropy caused by arrangement of $\{111\}\langle 101\rangle$ slip systems and the stress state.

\section{REFERENCES}

1) M. B. Henderson and J. W. Martin: Acta Metall. Mater. 44 (1996) 111126.

2) P. A. S. Reed, X. D. Wu and I. Sinclair: Metall. Mater. Trans. A, 31A (2000) 109-123.

3) K. Sugimoto, T. Sakaki, T. Horie, K. Kuramoto and O. Miyagawa: Metall. Trans. A, 16A (1985) 1457-1465.

4) K. Kakehi: Metall. Mater. Trans. A, 31A (2000) 421-430.

5) K. Kakehi: Scr. Mater. 42 (2000) 197-202.

6) N. K. Arakere and G. Swanson: Proc. of ASME TURBOEXPO 2000, May 8-11, Munich Germany, (2000) pp. 1-16.

7) K. Kakehi: Metall. Mater. Trans. A, 30A (1999) 1249-1259.

8) D. Bettge and W. Österle: Scr. Mater. 40 (1999) 389-395.

9) J. Telesman and L. J. Ghosn: Superalloys 1988, ed. by D. N. Duhl, G. Maurer, S. Antolovich, C. Lund and S. Reichman, (TMS, Warrendale, PA, 1988) pp. 615-624.

10) M. Okazaki, T. Tanabe and S. Nohmi: Metall. Trans. A 21A (1990) 2201-2208.

11) D. A. Koss and K. S. Chan: Acta Metall. 28 (1980) 1245-1252. 